Supporting Information for:

\title{
LC-MSn Methods for Saccharide Characterization of Monoglycosyl Flavonoids Using Post-Column Manganese Complexation
}

\author{
Barry D Davis and Jennifer S Brodbelt* \\ Department of Chemistry and Biochemistry, \\ University of Texas at Austin, \\ Austin, TX 78712
}

This section contains five figures consisting of supplemental mass spectral data that further demonstrate the use of manganese complexation to characterize flavonoid glycosides in standard solutions and in onion and apple extracts. These figures are referred to in the main text by the names $S-1$ through $S-5$. 


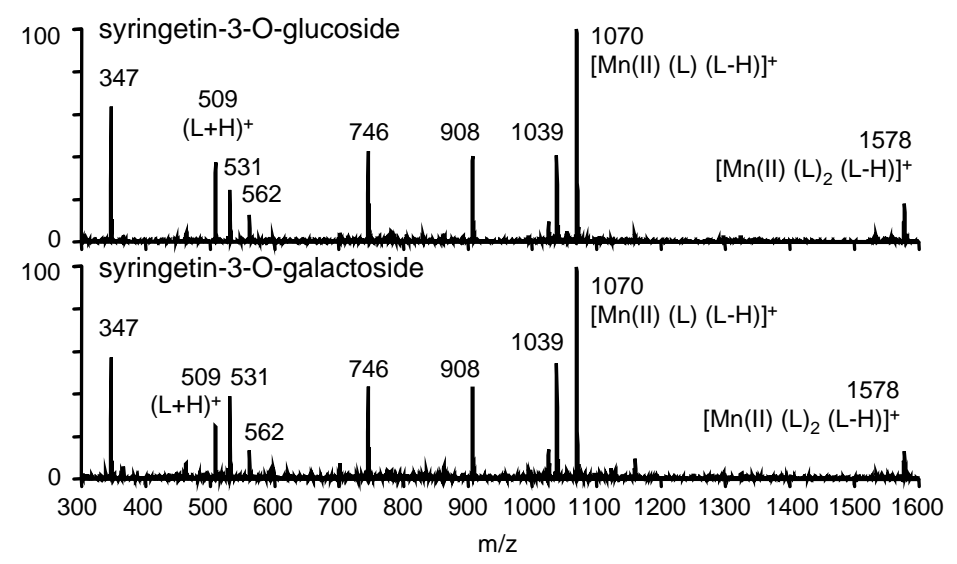

Figure S-1. Full scan spectra of selected flavonoid glycosides (L) with $\mathrm{Mn}(\mathrm{II})$. Ions are identified as 347: $(\mathrm{L}+\mathrm{H})^{+}-$hexose moiety; 531: $(\mathrm{L}+\mathrm{Na})^{+} ; 562$ : [Mn(II) (L$\mathrm{H})]^{+}$; 746: $[\mathrm{Mn}(\mathrm{II})(\mathrm{L})(\mathrm{L}-\mathrm{H})]^{+}-2$ hexose moieties; 908: $[\mathrm{Mn}(\mathrm{II})(\mathrm{L})(\mathrm{L}-\mathrm{H})]^{+}-$hexose moiety; 1039: $(2 \mathrm{~L}+\mathrm{Na})^{+}$.

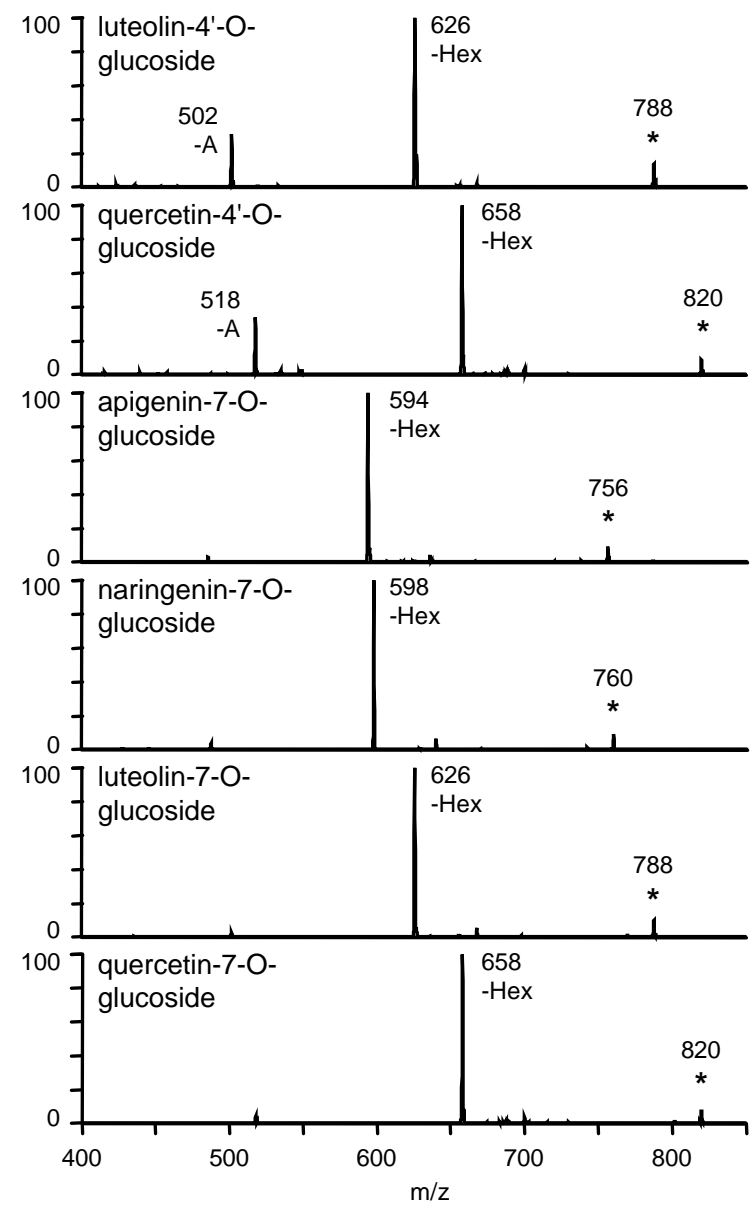

Figure S-2. $\mathrm{MS}^{3}$ spectra of $[\mathrm{Mn}(\mathrm{II})(\mathrm{L})(\mathrm{L}-\mathrm{H})]^{+}$ complexes of 4'-O-glucosides and 7-O-glucosides following the loss of one glucose moiety. The parent ion is denoted by an asterisk $\left(^{*}\right)$. Fragment ions are labeled as: -Hex (loss of a hexose moiety), -A (loss of an aglycon portion). The CAD energies ranged from $21-26.5 \%$.

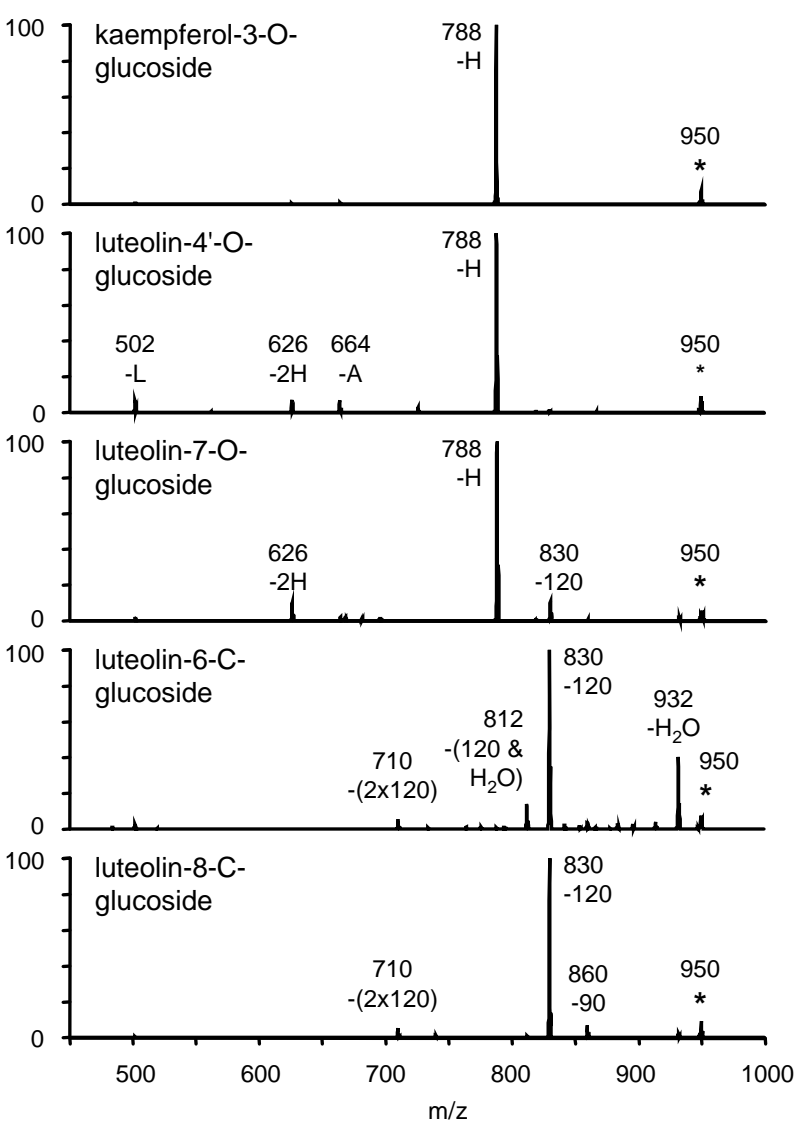

Figure S-3. CAD spectra of [Mn(II) (L) (L-H)] $]^{+}$ complexes of isomeric flavonoid glycosides. Fragment ions are labeled as $-\mathrm{H}$ (loss of a hexose residue), $-2 \mathrm{H}$ (loss of two hexoses residues), -A (loss of an aglycon portion), -L (loss of one flavonoid glycoside molecule), -120 (0,2 cross-ring saccharide cleavage), -90 (0,3 cross-ring saccharide cleavage). 


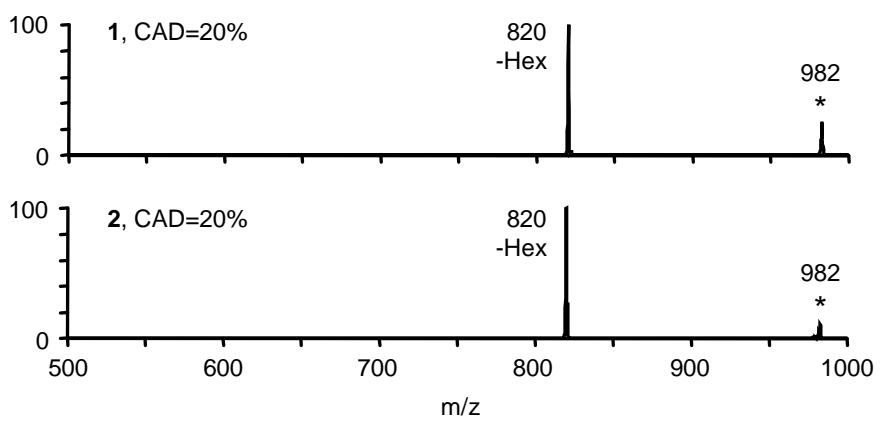

Figure S-4. MS/MS spectra of Mn complexes involving components of the Fuji apple peel extract. The parent ion, [Mn(II) $(\mathrm{L})(\mathrm{L}-\mathrm{H})]^{+}$, is denoted by an asterisk(*). Fragment ions are labeled as $-\mathrm{Hex}$ (loss of a hexose moiety).
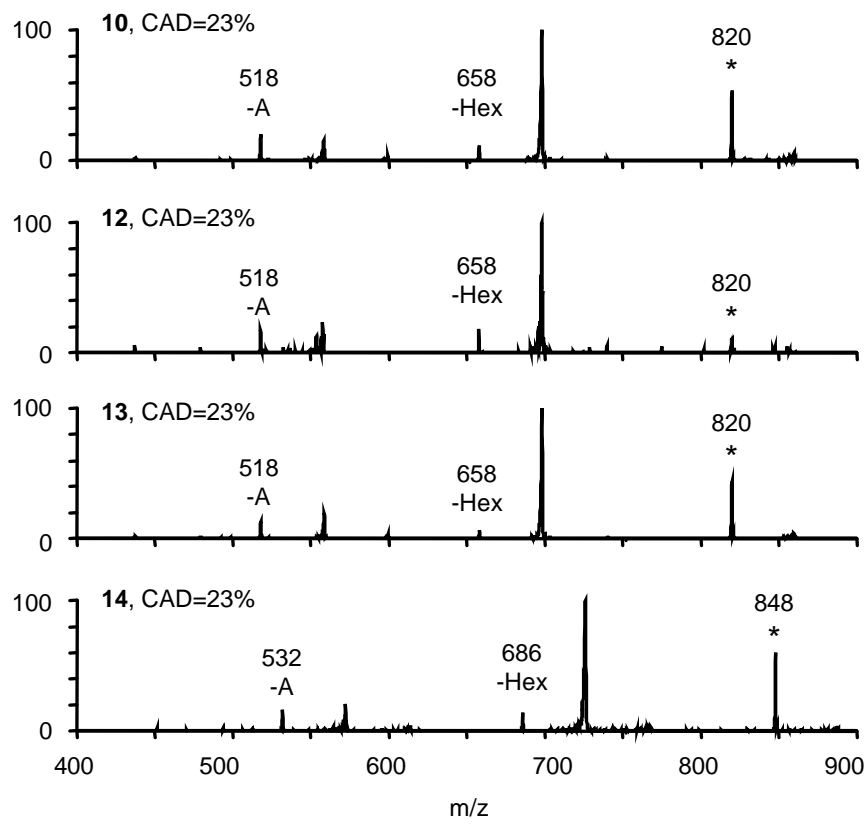

Figure S-5. MS ${ }^{3}$ spectra of $\mathrm{Mn}$ complexes involving components of the red onion extract. The parent ion is denoted by an asterisk( $\left.{ }^{*}\right)$. Fragment ions are labeled as - Hex (loss of a hexose moiety), -A (loss of an aglycon portion). Some of the additional peaks are due to post-CAD adduction of acetonitrile $(+41 \mathrm{u})$. 